\title{
COMMISSION 28: GALAXIES
}

\author{
(GALAXIES)
}

PRESIDENT: F. Bertola

VICE-PRESIDENT: S. Okamura

ORGANIZING COMMITTEE: C. Balkowski, D. Burstein, S. Chakrabarti, P. de Zeeuw, M. Feast, J. Huchra, V. Trimble, R. Wielebinsky, A. Zasov \&

Z.-L. Zou

\section{INTRODUCTION}

This report covers the period July 1996 to June 1999. It has been prepared by the President of the Commission with contributions from the members of the Organizing Committee and Dr. E.M. Corsini. As discussed in Kyoto and decided by the Organizing Committee, the report is meant to be in the "short" version.

\section{SELECTED TOPICS}

\subsection{Formation and Evolution, Structure, Kinematics and Dynamics (P.T. de Zeeuw)}

The realization by Steidel and others that one can select the Lyman break galaxies photometrically, and then follow up spectroscopically, opened up the study of high redshift normal galaxies: it is now easy to find them, so that theories can finally be tested.

The Madau diagram of the star formation history of the Universe.

Pushing the redshift barrier further: Franx's discovery of the $z=4.92$ lensed arc, which allows imaging of this high $z$ object with unprecedented spatial resolution. Further detection of objects beyond $z=5$.

Resolved kinematics of galaxies out to a redshift of about 1, with the systematic measurement of the fundamental plane as function of $z$ by van Dokkum and collaborators as an excellent example.

Determination of masses of central black holes in normal galaxies by reliable techniques (e.g. van der Marel et al. 1997), with as result the consensus that many, if not all, normal galaxies have a central black hole, but much discussion on the possible relation with the global properties of the parent galaxy. The recent meeting in Garching in honour of Giacconi made clear that there is much scatter in the black hole mass at fixed luminosity of the parent bulge.

The coming of age of integral field spectroscopy, following on the pioneering work of the French group with TIGER. OASIS measurements of the asymmetric nucleus of M31 are quite remarkable (Bacon)

The HST study by Carollo and collaborators of the nuclei of spiral bulges, which reveal much structure, tightly wound arms, distinct nuclei, and signs of recent star formation, even in bulges which, from the ground, appear featureless.

The MACHO and related projects resulting in 20 events towards the Large Magellanic Cloud (LMC), and lively debate on the nature of the machos; a large fraction may well be low-mass stars in the LMC stellar halo. A large mass range of halo objects is thereby ruled out. 


\subsection{Fundamental Parameters (Classification, Colors, Luminosities, Masses, Radii, Luminosity and Mass Functions) (S. Okamura)}

Classification Towards the era of large and/or deep imaging and redshift surveys, quantitative morphological classification based on photometric parameters (Abraham et al. 1996; Marleau \& Simard 1998; Okamura et al. 1999), detection of structural components (Andreon \& Davoust 1997), and artificial neural network (Odewahn et al. 1996; Owens et al. 1996) have been studied in addition to conventional eye inspection (Huang et al. 1998). Hubble sequence requires modification and/or extension to incorporate morphologies encountered at high redshifts and at low luminosity (van den Bergh 1997). Quantifying peculiarity and irregularities of galaxy images (Naim et al. 1997b; Takamiya 1999) may be a key (Naim et al. 1997a). Classification of galaxy spectra has been successfully attempted using the principal component analysis (Folkes et al. 1996; Sodré \& Cuevas 1997; Galaz \& De Lapparent 1998; Glazebrook et al. 1998; Ronen et al. 1999 Connoly \& Szlay 1999).

Structural Parameters and Scaling Relations Study of the evolution of fundamental structural parameters of galaxies and their scaling relations out to $z z 1$ is now within our reach (Vogt et al. 1997; Roche et al. 1998; Lilly et al. 1998; Bouwens et al. 1998; Simard et al. 1999). Local samples to be used as the fiducial are not yet very extensive (de Jong 1996), and knowledge of low surface brightness galaxies is growing (Impey et al. 1996; Bothun et al. 1997; Dalcanton et al. 1997; O'Neil et al. 1997; Sprayberry et al. 1997; Impey \& Bothun 1997; Morshidi-Esslinger et al. 1999).

The fundamental plane of early-type galaxies provides constraints on theories of their formation and evolution and also gives a useful distance tool. Universality is a critical issue (Kelson et al. 1997; Bender et al. 1998; van Dokkum et al. 1998; Scodeggio et al. 1998; Pahre et al. 1998; Mobasher et al. 1999).

Luminosity Function As the luminosity function (LF) is probed deeper, the assumption that the general LF follows a Schechter function seems no longer tenable. Large numbers of dwarfs are present in nearby clusters and groups which make the slope $\alpha=d N / d L$ of the general LF sometimes as steep as -2 for $M_{R}>-14$ (Trentham 1997; de Propris \& Pritchet 1998; Hunsberger et al. 1998). The general LF of local field galaxies also shows a hint of a steepening in the faint end (Zucca et al. 1997; Trentham 1998), but most data do not reach enough depth to confirm it (Gaidos 1997; Valotto et al. 1997). The LF shape of local field galaxies depends on morphology (Marzke et al. 1997, 1998; Bromley et al. 1998; Metcalfe et al. 1998). Thus the shape of type-specific LF and the evaluation of type mix are critical issues of any assessment of the general LF. The normalization of the local LF remains uncertain by about a factor of 2 for $M_{B}<-17$, where the LF shape is well defined (Ratcliffe et al. 1998). Evolution of type-specific LF at intermediate redshifts $(0.2<z<1)$ is beginning to be witnessed both in fields (Lin et al. 1997, 1999; Heyl et al. 1997; Small et al. 1997; Liu et al. 1998) and possibly in clusters (Wilson et al. 1997).

Color-Magnitude Relation Color-magnitude relation of elliptical galaxies and its scatter has been used to constrain epoch of their formation (Ellis et al. 1997; Stanford et al. 1998; Bower et al. 1998; Kodama et al. 1998, 1999).

\subsection{Super Massive Black Holes in Galactic Nuclei (E.M. Corsini)}

In these years the number of convincing detections of supermassive black holes (SMBHs) in galactic nuclei has considerably increased, due to interesting improvements of the observational and interpretative tools. In 1995, Kormendy \& Richstone listed 8 objects in their review paper on this subject. Now, 20 galaxies (Ho 1998) are known to harbor SMBHs, and their masses have been determined with a variety of techniques. They include optical observations of stellar kinematics (remarkable are the ground-based data obtained by the Canada-France-Hawaii Telescope) and of ionized-gas kinematics (measured by HST Faint 
Object Spectrograph and Space Telescope Imaging Spectrograph), radio observations of water masers (done by Very Long Baseline Interferometry and Array), and monitoring of time variability of broad double-peaked Balmer lines.

The demographics of SMBHs suggests that they are a normal feature of the nuclei of bright galaxies, at least of those with a spheroidal component (i.e., ellipticals, S0's and bulged spirals). In fact there is no evidence for SMBHs in late-type spirals without bulge or in low-surface brightness galaxies to date. This relationship between SMBHs and spheroids offers some clues to their formation and evolution (e.g., the transition of triaxial galaxies toward more axisymmetric shapes induced by a nuclear black hole).

The SMBH masses scale with the masses (or equivalently with the luminosities) of their parent spheroids. This correlation is affected either by a considerable scatter $(\sim 2$ dex $)$ of the measured black hole masses at a given spheroid luminosity and by selection bias (e.g., it is difficult to detect small SMBHs in giant galaxies with low central surface brightness). Within these uncertainties, the detected SMBHs are in the correct mass range to explain the fueling and the evolution of quasars.

The state-of-the-art achievements in this field can be found in the following proceedings: The Central Regions of the Galaxy and Galaxies, IAU Symposium 184 (1998, edited by Sofue), and Black Holes in Binaries and Galactic Nuclei, ESO Workshop (1999, edited by Kaper et al.). The most valuable and up-to-date paper reviews about SMBHs include those by Ho (1998), Richstone et al. (1998), Merritt (1999) and van der Marel (1999). Each of them develops a different topic related to SMBHs (e.g., demographics, formation, co-evolution of black holes and host galaxies).

\subsection{Stellar Content (D. Burstein)}

The basic questions challenging our understanding of the stellar population of other galaxies remain: What are the distributions of age and elemental abundances as a function of galaxy morphological type and luminosity? What physics relates the dynamics of galaxies so tightly to their stellar populations (e.g., tight Tully-Fisher and fundamental plane relationships)? Most importantly, what physics determines the stellar initial mass function? Progress on addressing the first two of these questions was made on several fronts, including: $H S T$ high angular resolution studies of stellar populations in nearby galaxies and their clusters; $H S T$ and ground-based near-IR studies of these and other stellar populations; detailed studies of the stellar populations in the halo, bulge and nucleus of our own galaxy; better theoretical understandings of the late stages of stellar evolution, which in turn determine the kinds of hot stars we see in old stellar populations. Unfortunately, little progress was made in better understanding the physics of the initial mass function of stars.

\subsection{Interstellar Medium (R. Wielebinsky)}

The ISO satellite has given us a new insight in the far infrared emission in the Galaxy and galaxies. It fills an important gap in the spectral range. Also the X-ray satellites ROSAT, $A S C A$, and $B E P P O-S A X$ have given new insights into the interstellar medium (ISM). The shadowing of clouds in X-rays has become a new method of investigation. Several new $\mathrm{H}$ I surveys have become available: the Dwingeloo northern sky survey and the Parkes multibeam survey of selected areas of the southern sky. The ISM at $\mathrm{mm}$ and sub-mm wavelengths became accessible to observations both in continuum and in the many higher excitation lines. Telescopes on new high altitude sites have made this new area of research possible. Sensitive surveys of the whole sky in $\mathrm{H} \alpha$ are in progress, expected to give a new dimension to our knowledge of the ISM. Last but not least studies of magnetic fields in galaxies and in the Milky Way using radio polarization methods gave us additional information on this 'invisible' ISM. 


\subsection{Local Group (M.W. Feast)}

At the present time our knowledge of the detailed stellar content of the Local Group of Galaxies is being expanded rapidly, due mainly to improved instrumental facilities. In the Magellanic Clouds a very significant part of this expansion has resulted as a by-product of microlensing experiments. The results now being obtained are fundamental for our understanding of the formation and evolution of galaxies as well as for firmly establishing the extragalactic distance scale.

Studies of objects in the Local Group are well covered in two IAU symposia held in 1998: New Views on the Magellanic Clouds, IAU Symposium 190 (1999, edited by Chu et al.) and The Stellar Content of Local Group Galaxies, IAU Symposium 192 (1999, edited by Whitelock \& Cannon).

The following proceedings of recent meetings are also relevant: The Magellanic Clouds and other Dwarf Galaxies, Bonn/Bochum-Graduiertenkolleg Workshop (1998, edited by Richtler \& Braun), Stellar Astrophysics for the Local Group, VIII Canary Islands Winter School of Astrophysics (1998, edited by Aparicio et al.), H I in the Local Universe, Australia Telescope National Facility Workshop (1997, edited by Staveley-Smith), Dwarf Galaxies and Cosmology, XVIII Moriond Astrophysics Meeting (1999, edited by Thuan et al.). It is anticipated that the forthcoming book by van den Bergh (1999), The Galaxies of the Local Group, will provide a useful summary of the whole field.

\subsection{Active Galaxies (BL Lacs, Quasars, Seyfert Galaxies, Compact Galaxies, Starburst Galaxies) (Z.L. Zou)}

The broad iron $\mathrm{K} \alpha$ line, characterized by the line from a relativistic accretion disk around a black hole, is found quite common among Seyfert galaxies (Nandra et al. 1997) during past years. Disk line fits to the line profiles yield a face-on disk for Seyfert 1 galaxies and intermediate inclinations for X-ray-bright narrow emission line galaxies (NLXGs) when proper models are adopted (Weaver \& Reynolds 1998), consistent with the unification scheme. In a number of cases, where high quality data were available, the models require a rotating black hole (Iwasawa et al. 1996). The line intensities and profiles are variable on rather short time scale of order $10^{4} \mathrm{~s}$ (Yaqoob et al. 1996) in complicated patterns. The line intensity can either increase or decrease with continuum flux, or without continuum variation (Wang et al. 1999). The latter authors explained this as either complicated ionization dependence of line emissivity or due to the change in the illumination law. In some extreme states (NGC 4151 in highest state and MCG 6-30-15 during deep minimum), the line is extremely broad and strong, with most emission line from within 10 gravitational radii (Dabrowski et al. 1997).

On the theoretical aspect, the Advection-Dominated Accretion Flow (ADAF) becomes the most successful model for the low level activity in galactic center, including LINERs. The model can reproduce the broad band spectrum of these objects and explains the low efficiency of mass-energy conversion (Narayan et al. 1997); Turning to the Blazars, it is found that the broad band spectrum consists of bumps, peaking at optical/UV and at hard X-ray/gamma ray. The catalog of blazars seen by EGRET includes 51 sources (Mukherjee et al. 1997). The fluxes, as well as the energies, of these two peaks, are correlated. The low energy bump is now thought due to the synchrotron emission in the relativistic jets, while the high energy peak is produced by inverse Compton scattering (Xie et al. 1997). However, detailed physics about emission from jets has yet to be worked out. New constraints on the model come from the detection delays among different bands/frequencies (e.g. PKS 2155-304, Urry et al. 1997).

Monitoring the line and continuum variability in the Seyfert galaxies has been undertaken for nearly ten years up to now. While most previous results indicate no measurable delays among different continuum bands, the long, continuous observations of NGC 7469 by IUE and ground optical telescopes in 1997 reveal a wavelength dependent delays among the continuum at different wavelengths (Peterson et al. 1998). These measured delays are 
consistent with the prediction of an X-ray irradiation disk model. With the sizes of average line emission regions for more lines measured in NGC 5548 (Peterson et al. 1999), it is found that the velocity dispersions of broad line regions (BLRs) are well following the Keplerian law, this is considered as evidence for gravitationally bounded BLR and provide a reliable measure of mass within it. New record of QSO's redshift goes up to 5.0 (Fan et al. 1999). A catalogue of quasars and active nuclei (8th edition) contains 11,358 quasars, 357 BL Lac objects and 3,334 active galaxies (Veron-Cetty \& Veron 1998). Recent books on AGN include those of Krolik (1999), Peterson (1997) and Robson (1996). Many interesting reviews include those of luminous infrared galaxies by Sanders \& Mirabel (1996) starburst galaxies by Moorwood (1996), variability of active galactic nuclei (AGN) by Ulrich et al. (1997), and star formation in galaxies by Kennicutt (1998).

Proceedings of conferences on AGN include those of Quasar Hosts, ESO/IAC Conference (1997, edited by Clements and Perez-Fournon), Mass Ejection from AGN, Carnegie Observatories Workshop (1997, edited by Arav et al.) and Emission Lines in Active Galaxies: New Methods and Techniques, IAU Colloquium 159 (edited by Peterson et al.).

\subsection{Clusters and Intergalactic Medium (C. Balkowski)}

By far the number of publications (120 papers) using X-rays observation of clusters with EINSTEIN, ASCA and ROSAT is the highest. The most important results among the cluster papers is the huge amount of multiwavelength data obtained these last four years on nearby and distant clusters.

Many individual nearby and distant clusters have been studied combining X-ray and optical observations, many examples of merging between clusters have been suggested and numerical simulations have been done (Vikhlinin et al. 1997; Roettiger et al. 1997). Ebeling et al. (1996) have published a catalogue of 242 Abell clusters observed with ROSAT.

An investigation of cooling flows and general cluster properties from $\mathrm{X}$-ray image deprojection analysis of clusters of galaxies has shown that the X-ray luminosity, temperature and optical velocity dispersion relations depend significantly on the cooling flow mass-deposition rate, though with characteristic differences in the density profiles (White et al. 1997).

The properties of the intracluster medium in an ensemble of nearby galaxy clusters have been studied by Mohr et al. (1999).

The measurement of the elemental abundances has been done in four rich clusters (Mushotzky et al. 1996).

The X-ray luminosity function (Burns et al. 1996; Ebeling et al. 1997) has been obtained from ROSAT.

The luminosity-temperature relation obtained at $z=0.4$ with $A S C A$ data does not show any evolution (Mushotzky \& Scharf 1997), confirmed by Burke et al. (1997) with ROSAT data. The galaxy cluster luminosity-temperature relationship and iron abundances could be a measure of the formation history (Scharf \& Mushotzky 1997).

Many nearby clusters have been studied in detail by deep optical and infrared photometry, spectroscopy, among these Coma, Virgo and Shapley Ames concentration (Boselli et al. 1997; Bardelli et al. 1998).

Large samples of clusters (APM, CNOC, ENACS, EIS) have been studied (Dalton et al. 1997; Yee et al. 1998; Katgert et al. 1998; Olsen et al. 1999). The average mass profile of galaxy clusters has been derived and supports the hierarchical clustering theory for the formation of galaxy clusters in a cool collisionless, dark-matter-dominated universe (Carlberg et al. 1997). The implications of the fundamental plane parameters of clusters of galaxies derived from combined optical and X-ray data have been discussed by Fritsch $\&$ Buchert (1999). A large-scale bulk flow of galaxy clusters has been reported $90^{\circ}$ away from the flow found by Lauer \& Postman (Hudson et al. 1999). 
The study of very distant clusters have been possible using the HST and the Keck telescope (Lubin et al. 1998; Smail et al 1997; Ostrander et al. 1998; Oke et al. 1998; Malkan et al. 1996).

The influence of environment on the star formation rates of galaxies and the ButcherOemler effect has been studied in clusters at different redshifts (Hashimoto et al. 1998; Caldwell \& Rose 1997; Couch et al. 1998). According to Andreon \& Ettori (1999) the Butcher-Oemler effect is not formed from the same class of objects observed at different look-back times, selection effects could mimic the trends usually interpreted as changes due to evolution. The star formation histories in distant clusters has been analyzed by Poggianti et al. (1999).

Morphological transformation from galaxy harassment has been proposed by Moore et al. (1998). Numerical simulations show that most galaxies did not undergo morphological evolution (Martel et al. 1998).

The discrepancy between different cluster mass estimates via lensing phenomena and $\mathrm{X}$-ray measurements has been addressed by Wu et al. (1998) showing that the estimators may be safely applied on scales greater than the cores sizes. The viral discrepancy is reduced, on average, from a factor of 4 in the Newtonian analysis to a factor of 2 in the Modified Newton Dynamics analysis (Sanders 1999).

The Sunyaev-Zeldovich effect has been observed in many clusters, leading to an estimate of the Hubble constant of $54 \mathrm{~km} \mathrm{~s}^{-1} \mathrm{Mpc}^{-1}$ (Myers et al. 1997; Tsuboi et al. 1998). The first measurement of the submillimeter Sunyaev-Zeldovich effect has been reported (Lamarre et al. 1998).

A catalogue of galaxy cluster models have been proposed (van Kampen \& Katgert 1997).

\subsection{Interactions (A.V. Zasov)}

General Properties and Most Interesting Objects As a result of the statistical study of 540 edge-on galaxies, the images and preliminary statistical analysis of a sub-sample of 60 galaxies, that were selected to be S-type warped spirals were presented by Reshetnikov \& Combes (1999). Zaritsky \& Rix (1997) presented a measurement of lopsidedness for the stellar disks of 60 field spiral galaxies in terms of the azimuthal $m=1$ Fourier amplitude of stellar light. They showed that $\sim 30 \%$ of field spiral galaxies in a magnitude-limited sample exhibit significant lopsidedness at large radii ( $>1.5$ disk scale lengths). It was argued that this lopsidedness is caused by tidal interactions. An upper limit on the accretion rate of small galaxies was calculated. Waller et al. (1997) presented new evidence for the occurrence of tidal interactions in the disk of M101 during the last $10^{8}-10^{8} \mathrm{yr}$, based on the analysis of the deep far-ultraviolet image of this galaxy. Toledo et al. (1999) presented a statistical analysis of the optical emission properties for a mixed-morphology $(\mathrm{E}+\mathrm{S})$ sample of galaxy pairs. They found an average factor of 1.6 enhancement in the optical luminosity of the late-type pair components relative to an isolated galaxy control sample. This is interpreted as the optical signature of the interaction-star formation connection. Near-infrared spectra of 20 interacting galaxies from the Arp Catalog and the properties of similar galaxies for which only optical spectra are available were analyzed by Vanzi et al. (1998). They found excellent agreement between the types of activity determined from the infrared and optical, demonstrating that obscuration does not seriously bias the optical results. The molecular gas content of galaxies in Hickson groups was investigated by Leon et al. (1998) by means of $\mathrm{CO}$ observations. They found that the mass of molecular gas in compact group galaxies is similar to that in pairs and star burst samples.

Sotnikova \& Reshetnikov (1998) have made a detailed observational study and numerical simulation of the interacting system NGC 4676 (the Mice). They show that the kinematics of the tails is incompatible with the classical model of the Mice, which reproduces only the morphological features of the object. It is concluded that the galaxies of the system must have massive extended halos. A series of numerical experiments to study the 
evolution of galaxies through their encounters was performed by Funato \& Makino (1999). Their result suggests that when galaxies in a cluster evolve mainly through frequent encounters with each other, a relation $\sigma^{4}-M$ develops between the mass $M$ and the velocity dispersion $\sigma$ of the galaxies which is equivalent to the Faber-Jackson relation. Orbital decay of galactic satellites was considered by Cora et al. (1997). Their numerical results for low-mass satellites showed very good agreement with theoretical predictions. The structure of the galaxies is significantly altered during the orbital decay for satellite masses greater than 0.01 . The main mechanism involved in the satellite orbital decay is dynamical friction. A heating and thickening of the disk due to the merging of satellites was considered by Velazquez \& White (1999). Self-consistent N-body simulations they carried out showed that the results strongly depend on the orbit orientation. Disk galaxies may accrete quite massive satellites without destroying the disk, particularly, if the orbits are retrograde. A formation of shell structures was modeled by the detailed numerical simulations of the sinking of a gas-rich disk galaxy into a large elliptical (spherical) galaxy (Kojima \& Noguchi 1997). Particularly, it was shown that the resulting global distribution of the gas clouds is similar to that of the stars, though the latter make clearer shells. Thickening tilting or distortions in galactic disks, generated by the perturbation and accretion of satellites were modeled by numerical simulations and analyzed by Edelsohn \& Elmegreen (1997), Huang \& Carlberg (1997), and Sellwood et al. (1998). In particular, in the latter paper it was shown that the satellite does little damage to the disk until its decaying orbit resonantly excites large-scale disk bending waves.

Merging and Evolution of Interacting Systems Merging of galaxies of comparable masses and their consequent evolution was discussed in a series of works. A one-zone chemophotometric evolution model of disk-disk galaxy mergers was investigated by Shioya \& Bekki (1998) which applied their results to the explanation of the tight color-magnitude (C-M) relation in cluster ellipticals at low and intermediate redshift $(z<1)$. The origin of the C-M relation of elliptical galaxies was also considered by Bekki \& Shioya (1997). They especially investigate the dependence of the mean stellar metallicity of merger remnants on the rapidity of gas consumption by star formation during merging. Hibbard \& Yun (1999), using published luminosity and molecular gas profiles of the late-stage mergers NGC 3921, NGC 7252, and Arp 220, examined the expected luminosity profiles of the evolved merger remnants. The purpose of the study of Bekki (1998) was to explore dynamical effects of galaxy merging on gas replenishment from galactic halos. In particular, it was found that the galaxy merging greatly changes initial mass distribution of halo ISM of disk galaxies in such a way that final mean ISM density of the halo in a galaxy merger is considerably smaller compared with initial one.

\subsection{Distances and Redshifts (J.P. Huchra)}

In the last three years, progress in the study of large-scale structure and in the determination of the extragalactic distance scale has been enormous. Several major redshift data sets have been released, most notably the Las Campanas Redshift Survey with about 25,000 galaxy redshifts, the Center for Astrophysics (CfA) Survey, with about 18,000 redshifts and the Second Southern Sky Redshift Survey (SSRS2) with about 8,000. The CfA Redshift Catalogue now (9/99) contains about 130,000 galaxies and QSO's with redshifts, and the sky, with the exception of parts of the southern hemisphere galactic plane and the regions covered by the Magellanic Clouds, has pretty much been mapped to $m_{B} \sim 15$. Smaller, recently completed redshift surveys include the European Southern Observatory Slice Survey and the remainder of the original QDOT redshift survey, based on an IRAS 60 micron selected sample, now called the PSCz, which contains about 13,000 galaxies.

Several major new surveys are underway or just beginning. Large, dense surveys are being done with new multi-fiber spectrographs that allow the measurement of hundreds of galaxy redshifts simultaneously. The Two Degree Field (2dF) survey, using the 4-m Anglo Australian Telescope, has already amassed 50,000 new redshifts with a goal of obtaining 
250,000 galaxy and 20,000 QSO redshifts. The Sloan Digital Sky Survey (SDSS) is just now ramping up operations, and plans to observe $1,000,000$ galaxies over the northern celestial hemisphere using a catalog based on five color CCD imaging. A shallower, but full-sky galaxy redshift survey based on a 2 micron selected catalog from the 2 Micron All-Sky Survey (2MASS), is underway. Its goal is to measure first $\sim 125,000$ galaxies to a $\mathrm{K}_{S}$ limit of 12.2 , and to follow that up with a sparse sample to 13.5. The southern part of this survey is based on the planned 6DF survey, which will use a multi-fiber system on the SRC Schmidt. The 2MASS survey will produce the best map of the local galaxy density field. All of these surveys promise not only the ultimate in the determination of the clustering statistics of galaxies at low redshift, but also tremendous databases of spectral energy distributions and spectral types to use in the study of galaxy evolution.

Despite the results of our existing large surveys, key questions remain. Large-scale structure as observed is not yet matched in detail by the simulations. The real galaxy distribution tends to resemble surfaces rather than filaments, and the observed small-scale galaxy-galaxy velocity field (pairwise velocities) is very quiet, much more so than any existing simulation with reasonable cosmological parameters. The solution may lie in the improved data that surveys like SDSS will provide, but it may also lie in improved simulations with better input physics.

Since the refurbishment of the $H S T$, significant progress has been made on the precise measurement of nearby galaxy distances utilizing the Cepheid P-L relation and the superb spatial resolution of $H S T$. Two groups have been extremely active, the $H S T$ Key project on the Extragalactic Distance Scale, headed by W. Freedman, J. Mould and R. Kennicutt, and the SN Ia calibration team headed by A. Saha, A. Sandage and G. Tammann. Both teams have developed new calibrations of secondary and tertiary indicators that are used to measure distances to galaxies far enough out in the Hubble flow such that the determination of the Hubble Constant is not significantly affected by peculiar motions. Coupled with significant advances in the internal calibration of the secondary indicators, such as the SN Ia light-curve shape analysis, and more relative distance determinations using such high precision yardsticks as the Surface Brightness Fluctuation and $I$-band Tully-Fisher methods, the discrepancy between groups working on the local determination of $H_{0}$ is now at the $20 \%$ level rather than the factor of two level. Modulo the exact determination of the $\mathrm{P}-\mathrm{L}$ zero point (the accurate determination of the distance of the $\mathrm{LMC}$ ), $H_{0}$ is probably in the range $65 \pm 10 \mathrm{~km} \mathrm{~s}^{-1} \mathrm{Mpc}^{-1}$.

F. Bertola

President of the Commission

\section{References}

Abraham, R. G., et al. 1996, ApJS, 107, 1

Andreon, S. \& Davoust, E. 1997, A\&A, 319, 747

Andreon, S. \& Ettori, S. 1999, ApJ, 516, 647

Aparicio, A., Herrero, A. \& Sanchez, F. (eds.) 1998, Stellar Astrophysics for the Local Group, VIII Canary Islands Winter School of Astrophysics, (Cambridge: Cambridge University Press)

Arav, N., Shlosman, I. \& Weymann R. J. (eds.) 1997, Mass Ejection from AGN, Proc. Carnegie Observatories Workshop, (San Francisco: Astronomical Society of the Pacific)

Bardelli, S., Zucca, E., Zamorani, G., Vettolani, G. \& Scaramella, R. 1998, MNRAS, 296, 599

Bekki, K. \& Shioya, Y. 1997, ApJ, 486, 197

Bekki, K. 1998, A\&A, 334, 814

Bender, R., et al. 1998, ApJ, 493, 529 
Boselli, A., et al. 1997, A\&A, 324, L13

Bothun, G., Impey, C. \& McGaugh, S. 1997, PASP, 109, 745

Bouwens, R., Broadhurst, T. \& Silk, J. 1998, ApJ, 506, 557

Bower, R. G., Kodama, T. \& Terlevich, A. 1998, MNRAS, 299, 1193

Bromley, B. C., Press, W. H., Lin, H. \& Kirshner, R. P. 1998, ApJ, 505, 25

Burke, D. J., Collins, C. A., Sharples, R. M., Romer, A. K., Holden, B. P. \& Nichol, R. C. 1997, ApJ, 488, L83

Burns, J. O., et al. 1996, ApJ, 467, L49

Caldwell, N. \& Rose, J. A. 1997, AJ, 113, 492

Carlberg, R. G., et al. 1997, ApJ, 485, L13

Chu, Y.-H., Suntzeff, N., Hesser, H. \& Bohlender, B. (eds.) 1999, IAU Symp. 190, New Views on the Magellanic Clouds, (San Francisco: Astronomical Society of the Pacific), in press

Clements, D. L. \& Perez-Fournon, I. (eds.) 1997, Quasar Hosts, Proc. ESO/IAC Conference, (Berlin: Springer-Verlag)

Connolly, A. J. \& Szalay, S. 1999, AJ, 117, 2052

Cora, S. A., Muzzio, J. C. \& Vergne, M. M. 1997, MNRAS, 289, 253

Couch, W. J., Barger, A. J., Smail, I., Ellis, R. S. \& Sharples, R. M. 1998, ApJ, 497, 188

Dabrowski, Y., Fabian, A. C., Iwasawa, K., Lasenby, A. N. \& Reynolds, C. S. 1997, MNRAS, 288, L11

Dalcanton, J., Spergel, D. N., Gunn, J. E., Schmidt, M. \& Schneider, D. P. 1997, AJ, 114, 635

Dalton, G. B., Maddox, S. J., Sutherland, W. J. \& Efstathiou, G. 1997, MNRAS, 289, 263

De Jong, R.S. 1996, A\&A, 313, 45

De Propris, R. \& Pritchet, C. J. 1998, AJ, 116, 1118

Ebeling, H., Voges, W., Bohringer, H., Edge, A. C., Huchra, J. P. \& Briel, U. G. 1996, MNRAS, 281, 799

Ebeling, H., Edge, A. C., Fabian, A. C., Allen, S. W., Crawford, C. S. \& Boehringer, H. 1997, ApJ, 479, L101

Edelsohn, D. J. \& Elmegreen, B. G. 1997, MNRAS, 287, 947

Ellis, R. S., et al. 1997, ApJ, 483, 582

Fan, X., et al. 1999, AJ, 118, 1

Folkes, S. R., Lahav, O. \& Maddox, S. J. 1996, MNRAS, 283, 651

Fritsch, C. \& Buchert, T. 1999, A\&A, 344, 749

Funato, Y. \& Makino, J. 1999, ApJ, 511, 625

Gaidos, E. J. 1997, AJ, 113, 117

Galaz, G. \& De L'apparent, V. 1998, A\&A, 332, 459

Glazebrook, K., Offer, A. R. \& Deeley, K. 1998, ApJ, 492, 98

Hashimoto, Y., Oemler, A., Jr., Lin, H. \& Tucker, D. L. 1998, ApJ, 499, 589

Heyl, J., Colless, M., Ellis, R. S. \& Broadhurst, T. 1997, MNRAS, 285, 613

Hibbard, J. E. \& Yun, M. S. 1999, ApJ, 522, L93

Ho, L. C. 1998, in Observational Evidence for Black Holes in the Universe, ed. S. K. Chakrabarti (Dordrecht: Kluwer), 157

Huang, J.-S., Cowie, L. L. \& Luppino, G. A. 1998, ApJ, 496, 31

Huang, S. \& Carlberg, R. G. 1997, ApJ, 480, 503

Hudson, M. J., Smith, R. J., Lucey, J. R., Schlegel, D. J. \& Davies, R. L. 1999, ApJ, 512, L79

Hunsberger, S. D., Charlton, J. C. \& Zaritsky, D. 1998, ApJ, 505, 536 
Impey, C. D., Sprayberry, D., Irwin, M. J. \& Bothun, G. D. 1996, ApJS, 105, 209

Impey, C. \& Bothun, G. 1997, ARA\&A, 35, 267

Iwasawa, K., et al. 1996, MNRAS, 282, 1038

Kaper, L., van den Heuvel, E. P. J. \& Woudt, P. A. (ed.) 1999 Black Holes in Binaries and Galactic Nuclei, Proc. ESO Workshop in honour of Prof. R. Giacconi (Berlin: Springer-Verlarg), in press

Katgert, P., Mazure, A., Den Hartog, R., Adami, C., Biviano, A. \& Perea, J. 1998, A\&AS, 129,399

Kelson, D. D., Van Dokkum, P. G., Franx, M., Illingworth, G. D. \& Fabricant, D. 1997, ApJ, 478, L13

Kennicutt, R. C., Jr. 1998, ARA\&A, 36, 189

Kodama, T., Arimoto, N., Barger, A. J. \& Aragon-Salamanca, A. 1998, A\&A, 334, 99

Kodama, T., Bower, R. G. \& Bell, E. F. 1999, MNRAS, 306, 561

Kormendy, J. \& Richstone, D. 1995, ARA\&A, 33, 581

Kojima, M. \& Noguchi, M. 1997, ApJ, 481, 132

Krolik, J. H. 1999, Active Galactic Nuclei: from the Central Black Hole to the Galactic Environment, (Princeton: Princeton University Press)

Lamarre, J. M., et al. 1998, ApJ, 507, L5

Leon, S., Combes, F. \& Menon, T. K. 1998, A\&A, 330, 37

Lilly, S., et al. 1998, ApJ, 500, 75

Lin, H., Yee, H. K. C., Carlberg, R. G. \& Ellingson, E. 1997, ApJ, 475, 494

Lin, H., et al. 1999, ApJ, 518, 533

Liu, C. T., Green, R. F., Hall, Patrick B. \& Osmer, P. S. 1998, AJ, 116, 1082

Lubin, L. M., et al. 1998, AJ, 116, 584

Malkan, M. A., Teplitz, H. \& McLean, I. S. 1996, ApJ, 468, L9

Marleau, F. R. \& Simard, L. 1998, ApJ, 507, 585

Martel, H., Premadi, P. \& Matzner, R. 1998, ApJ, 497, 512

Marzke, R. O. \& Da Costa, L. N. 1997, AJ, 113, 185

Marzke, R. O., Da Costa, L. N., Pellegrini, P. S., Willmer, C. N. A. \& Geller, M. J. 1998, ApJ, 503, 617

Merritt, D. 1999, in Galaxy Dynamics, ed. D. Merritt, J. A. Sellwood \& M. Valluri, Proc. Rutgers Conference, ASP Conf. Series 182, (San Francisco: Astronomical Society of the Pacific)

Metcalfe, N., Ratcliffe, A., Shanks, T. \& Fong, R. 1998, MNRAS, 294, 147

Mobasher, B., Guzman, R., Aragon-Salamanca, A. \& Zepf, S. 1999, MNRAS, 304, 225

Mohr, J. J., Mathiesen, B. \& Evrard, A. E. 1999, ApJ, 517, 627

Morshidi-Esslinger, Z., Davies, J. I. \& Smith, R. M. 1999, MNRAS, 304, 297

Moore, B., Lake, G. \& Katz, N. 1998, ApJ, 495, 139

Moorwood A. F.M. 1996, SSRv, 77, 303

Mukherjee, R., et al. 1997, ApJ, 490, 116

Mushotzky, R., et al. 1996, ApJ, 466, 686

Mushotzky, R. F. \& Scharf, C. A. 1997, ApJ, 482, L13

Myers, S. T., Baker, J. E., Readhead, A. C. S., Leitch, E. M. \& Herbig, T. 1997, ApJ, 485, 1

Naim, A., Ratnatunga, K. U. \& Griffiths, R. E. 1997a, ApJS, 111, 357

Naim, A., Ratnatunga, K. U. \& Griffiths, R. E. 1997b, ApJ, 476, 510

Nandra, K., George, I. M., Mushotzky, R. F., Turner, T. J. \& Yaqoob, T. 1997, ApJ, 477, 602 
Narayan, R., Barret, D. \& McClintock, J. E. 1997, ApJ, 482, 448

Odewahn, S. C., Windhorst, R. A., Driver, S. P. \& Keel, W. C. 1996, ApJ, 472, L13

Okamura, S., Yasuda, N., Shimasaku, K., Yagi, M. \& Weinberg, D. H. 1999, PASP, 111, 31

Oke, J. B., Postman, M. \& Lubin, L. M. 1998, AJ, 116, 549

Olsen, L. F., et al. 1999, A\&A, 345, 363

O'Neil, K., Bothun, G.D., Schombert, J., Cornell, M. E. \& Impey, C.D. 1997, AJ, 114, 2448

Ostrander, E. J., Nichol, R. C., Ratnatunga, K. U. \& Griffiths, R. E. 1998, AJ, 116, 2644

Owens, E. A., Griffiths, R.E. \& Ratnatunga, K. U. 1996, MNRAS, 281, 153

Pahre, M. A., De Carvalho, R. R. \& Djorgovski, S. G. 1998, AJ, 116, 1606

Peterson, B. M. 1997, An Introduction to Active Galactic Nuclei, (Cambridge: Cambridge University Press)

Peterson, B. M., Cheng, F.Z. \& Wilson, A. S. (eds.) 1997, IAU Coll. 159, Emission Lines in Active Galaxies: New Methods and Techniques, (San Francisco: Astronomical Society of the Pacific)

Peterson, B. M., et al. 1998, PASP, 110, 660

Peterson, B. M., et al. 1999, ApJ, 510, 659

Poggianti, B. M., et al. 1999, ApJ, 518, 576

Ratcliffe, A., Shanks, T., Parker, Q. A. \& Fong, R. 1998, MNRAS, 293, 197

Reshetnikov, V. \& Combes, F. 1999, A\&AS, 138, 101

Richstone, D. et al. 1998, Nature, 385, A14

Richtler, T. \& Braun, J.M. (eds.) 1998, The Magellanic Clouds and Other Dwarf Galaxies,

Proc. Bonn/Bochum-Graduiertenkolleg Workshop, (Aachen: Shaker Verlag)

Robson, I. 1996, Active Galactic Nuclei, (New York: Wiley)

Roche, N., Ratnatunga, K., Griffiths, R. E., Im, M. \& Naim, A. 1998, MNRAS, 293, 157

Roettiger, K., Burns, J. O. \& Loken, C. 1996, ApJ, 473, 651

Ronen, S., Aragon-Salamanca, A. \& Lahav, O. 1999, MNRAS, 303, 284

Sanders, D. B. \& Mirabel, I. F. 1996, ARA\&A, 34, 749

Sanders, R. H. 1999, ApJ, 512, L23

Scharf, C. A. \& Mushotzky, R. F. 1997, ApJ, 485, L65

Scodeggio, M., Giovanelli, R. \& Haynes, M. P. 1998, AJ, 116, 2738

Sellwood, J. A., Nelson, R. W. \& Tremaine, S. 1998, ApJ, 506, 590

Shioya, Y. \& Bekki, K. 1998, ApJ, 504, 42

Simard, L., et al. 1999, ApJ, 519, 563

Sodre, L. \& Cuevas, H. 1997, MNRAS, 287, 137

Smail, I., et al. 1997, ApJS, 110, 213

Small, T. A., Sargent, W. L. W. \& Hamilton, D. 1997, ApJ, 487, 512

Sofue, Y. (ed.) 1998, IAU Symp. 184, The Central Regions of the Galaxy and Galaxies (Dordrecht: Kluwer)

Sotnikova, N. Y. \& Reshetnikov, V. P. 1998, AstL, 24, 73

Sprayberry, D., Impey, C. D., Irwin, M. J. \& Bothun, G. D. 1997, ApJ, 482, 104

Stanford, S. A., Eisenhardt, P. R. \& Dickinson, M. 1998, ApJ, 492, 461

Staveley-Smith, L. (ed.) 1997, $\mathrm{H}_{\mathrm{I}}$ in the Local Universe, Proc. Australia Telescope National Facility Workshop, PASA, 14,

Takamiya, M. 1999, ApJS, 122, 109

Thuan, X., Balkowski, C., Cayatte, V. \& Tran Thanh Van, J. (eds.) 1999, Dwarf Galaxies and Cosmology, XVIII Moriond Astrophysics Meeting, (Gif-sur-Yvette: Editions Frontières), in press 
Toledo, H. M. H., Dultzin-Hacyan, D., Gonzalez, J. J. \& Sulentic, J. W. 1999, AJ, 118, 108 Trentham, N. 1997, MNRAS, 286, 133

Trentham, N. 1998, MNRAS, 294, 193

Tsuboi, M., Miyazaki, A., Kasuga, T., Matsuo, H. \& Kuno, N. 1998, PASJ, 50, 169

Ulrich, M.-H., Maraschi, L. \& Urry, C. M. 1997, ARA\&A, 35, 445

Urry, C. M., et al. 1997, ApJ, 486, 799

Valotto, C. A., Nicotra, M. A., Muriel, H. \& Lambas, D. G. 1997, ApJ, 479, 90

Van Den Bergh, S. 1997, AJ, 113, 2054

Van Den Bergh, S. 1999, The Galaxies of the Local Group, (Cambridge: Cambridge University Press)

Van Der Marel, R. P., De Zeeuw, P., Rix, H.-W. \& Quinlan, G. D. 1997, Natur, 385, 610

Van Der Marel, R. P. 1999, in IAU Symp. 186, Galaxy Interactions at Low and High Redshift ed. J. Barnes \& D. B. Sanders (Kluwer: Dordrecht)

Van Dokkum, P. G., Franx, M., Kelson, D. D. \& Illingworth, G. D. 1998, ApJ, 504, L17

Van Kampen, E. \& Katgert, P. 1997, MNRAS, 289, 327

Vanzi, L., Alonso-Herrero, A. \& Rieke, G. H. 1998, ApJ, 504, 93

Velazquez, H. \& White, S. D. M. 1999, MNRAS, 304, 254

Veron-Cetty, M.-P. \& Veron, P. 1998, A Catalogue of Quasars and Active Nuclei, (Garching bei München: European Southern Observatory)

Vikhlinin, A., Forman, W. \& Jones, C. 1997, ApJ, 474, L7

Vogt, N. P., et al. 1997, ApJ, 479, L121

Waller, William H., et al. 1997, ApJ, 481, 169

Wang, J. X., Zhou, Y. Y., Xu, H. G. \& Wang, T. G. 1999, ApJ, 516, L65

Weaver, K. A. \& Reynolds, C. S. 1998, ApJ, 503, L39

White, D. A., Jones, C. \& Forman, W. 1997, MNRAS, 292, 419

Whitelock, P. \& Cannon, R. (eds.) 1999, IAU Symp. 192, The Stellar Content of Local Group Galaxies, (San Francisco: Astronomical Society of the Pacific)

Wilson, G., Smail, I., Ellis, R. S. \& Couch, W. J. 1997, MNRAS, 284, 915

Wu, X.-P., Chiueh, T., Fang, L.-Z. \& Xue, Y.-J. 1998, MNRAS, 301, 861

Xie, G. Z., Zhang, Y. H. \& Fan, J. H. 1997, ApJ, 477, 114

Yaqoob, T., Serlemitsos, P. J., Turner, T. J., George, I. M. \& Nandra, K. 1996, ApJ, 470, L27

Yee, H. K. C., Ellingson, E., Morris, S. L., Abraham, R. G. \& Carlberg, R. G. 1998, ApJS, 116,211

Zaritsky, D. \& Rix, H.-W. 1997, ApJ, 477, 118

Zucca, E., et al. 1997, A\&A, 326, 477 